\title{
Anabases
}

ANABASES Traditions et réceptions de l'Antiquité

$26 \mid 2017$

Varia

\section{Antiquités parallèles (7). La starlette des bandelettes}

\section{Claude Aziza}

\section{(2) OpenEdition}

Journals

Édition électronique

URL : http://journals.openedition.org/anabases/6363

DOI : 10.4000/anabases.6363

ISSN : 2256-9421

\section{Éditeur}

E.R.A.S.M.E.

\section{Édition imprimée}

Date de publication : 1 novembre 2017

Pagination : 177-181

ISSN : 1774-4296

\section{Référence électronique}

Claude Aziza, «Antiquités parallèles (7). La starlette des bandelettes », Anabases [En ligne], 26 | 2017, mis en ligne le 01 novembre 2020, consulté le 20 janvier 2021. URL : http://journals.openedition.org/ anabases/6363 ; DOI : https://doi.org/10.4000/anabases.6363 




Traditions et Réceptions de l'Antiquité

$$
\begin{aligned}
& N \circ 26 \\
& 2017
\end{aligned}
$$

\section{E.R.A.S.M.E.}

Université Toulouse - Jean Jaurès 



\section{Sommaire}

$\mathrm{N}^{\circ} 26-2017$

\section{Historiographie et identités culturelles}

Jocelyne Peigney

La polysémie poétique d'anabase chez Saint-John Perse :

évocations, étymologie et botanique $\ldots \ldots \ldots \ldots \ldots \ldots \ldots \ldots \ldots \ldots$

Nicolas FAELLI

Les références antiques des fondateurs de l'Amérique française

au XVII siècle ................................. I9

Silvia Alaura

Setting the stage for Hittite studies in Victorian England:

practices and methods of the 1870 . . . . . . . . . . . . .

\section{Traditions du patrimoine antique}

“ Restituer Herculanum I. Des archives de fouilles aux restitutions 3D »

Alexandra Dardenay et Adeline Grand-Clément

Restituer Herculanum au XXI ${ }^{\mathrm{e}}$ siècle. L'apport des archives. . . . . . . . 55

Antonella Coralini

In situ e alibi, dallo scavo integrato alla cultura dell'abitare :

Vesuviana-DHER a Ercolano . . . . . . . . . . . . . . . . 67 67

Agnes Allroggen-Bedel

I documenti settecenteschi come strumenti per lo studio

degli scavi ercolanesi . . . . . . . . . . . . . . . Io3

James ANDREws

Revisiting the upper floors of the casa di Argo at Herculaneum . . . . . . II7 
Nicolas MonteIx

Les archives des fouilles d'Herculanum au XX $\mathrm{XX}^{\mathrm{e}}$ siècle :

analyse critique et pistes d'exploitation . . . . . . . . . . I43

\section{Archéologie des savoirs}

Yannick Le PAPE

L'inspiration et l'imprudence. Poésie de l'anticomanie

dans la critique d'art du second XIX ${ }^{\mathrm{e}}$ siècle . . . . . . . . . . . . . I57

\section{Actualités et débats}

Claude AzızA

Antiquités parallèles (7). La starlette des bandelettes . . . . . . . . . I77

Alexandra TrachseL

La réception d'Homère dans le domaine de la rhétorique $\ldots \ldots \ldots$. . . I83

Claude Aziza

Curiosa (2) In vino (vin/eau) veritas (very tasse) : drame de l'ivresse . . . . I89

\section{Lire, relire la bibliothèque des sciences de l'Antiquité}

Laurent OLIVIER

Pour en finir avec la race : Henri Hubert et l'anthroposociologie

À propos du commentaire de “L'Aryen, son rôle social », de Vacher de Lapouge $(\mathrm{r} 899) \ldots \ldots \ldots$

Henri Hubert

Compte rendu de Georges VACher de LAPouge, L'Aryen, son rôle social, L'Année sociologique, igoo, p. i45-146 . . . . . . . . . . . . . . . . . 203

\section{L'atelier de l'histoire : chantiers historiographiques}

L'Atelier des doctorants (coordonné par Adeline Grand-Clément) (14)

Francesco LoDÀ,

L'initiation des guerriers chez les peuples indo-européens.

Une enquête historiographique dans les pas de Georges Dumézil . . . 207

\section{Comptes rendus}

Benjamin Anderson et Robert G. Ousterhout

Palmyra I885. The Wolfe Expedition and the photographs

of John Henry Haynes (M. Sartre) . . . . . . . . . . . . . . . . . . . 2 215 
Mary R. BAchvarova

From Hittite to Homer. The Anatolian Background of Ancient Greek Epic (G. Hoffmann) . . . . . . . . . . . . . . . . .

Patrick Baker, Ronny Kaiser, Maike Priesterjahn, Johannes Helmrath (éd.) Portraying the Prince in the Renaissance. The Humanist Depiction of Rulers in Historiographical and Biographical Texts (G. Demelemestre). . 220

Brigitte BAKHоUсHE (éd.)

Science et exégèse. Les interprétations antiques et médiévales du récit biblique de la création des éléments (Genèse I, I-8) (F. P. BARONE) . . . . . . .

Laurence Baurain-Rebillard (éd.)

Héros grecs à travers le temps : autour de Persée, Thésée, Cadmos et Bellérophon (C. Giovénal) . . . . . . . . . . . . . . . .

Mark W. PAdiLla,

Classical myth in four films of Alfred Hitchcock . . . . . . . . . . . . . . .

Vivien Bessieres

Le Péplum et après? L’Antiquité gréco-romaine dans les récits contemporains (Cl. Aziza) . . . . . . . . . . . . . . . . . 225

Pierre Briant

Alexandre. Exégèse des lieux communs (É. Guillon) . . . . . . . . . . . . . 227

Paulo ButTi DE Lima

Il piacere delle Immagini. Un tema aristotelico nella riflessione moderna sull'arte (C. Noacco) . . . . . . . . . . . . . . . . . . .

Bénédicte Coudì̀re

La truelle et le phylactère. La proximité des images (M. Scapin) . . . . . . . 23I

Thorsten Fögen, Richard WARREN (éd.)

Graeco-Roman Antiquity and the Idea of Nationalism in the rgth Century, Case Studies (M. Champier) . . . . . . . . . . . . . . . ${ }_{232}$

Ronald H. Fritze

Egyptomania. A History of Fascination, Obsession and Fantasy

(Fabien Bièvre-Perrin) . . . . . . . . . . . . . . . . . . . . . 234

Joseph Geiger

Hellenism in the East. Studies on Greek Intellectuals in Palestine

(C. Bonnet). . . . . . . . . . . . . . . . . 236

Tiffany Jenkins

Keeping their marbles. How the treasures of the past ended up in museums... and why they should stay there (C. Valenti) . . . . . . . . . . 238 
Ayelet H. Lushkov

Magistracy and the Historiography of the Roman Republic.

Politics in Prose (C. Landrea) . . . . . . . . . . . . . . . . . . . . . . 240

Jean-Claude MüHLEthaler

Énée le mal-aimé. Du roman médiéval à la bande dessinée

(Fl. Bouchet) ........................ 24I

Laurent PERNoT

Epideictic Rhetoric, Questioning the Stakes of Ancient Praise

(C.Psilakis) ......................... 243

Priscian

Answers to King Khosroes of Persia (O. Gengler) . . . . . . . . . . . . . . 245

Paul Robertson

Paul's letters and contemporary Greco-Roman literature:

theorizing a new taxonomy (M. Cambron-Goulet) . . . . . . . . . . . . . . 247

Federico Santangelo (éd.)

Approaching the Roman Revolution. Sir Ronald Syme,

Papers on Republican History (C. Landrea) . . . . . . . . . . . . . . . . . . 249

Molly Swetnam-BurLand

Egypt in Italy. Visions of Egypt in Roman Imperial Culture

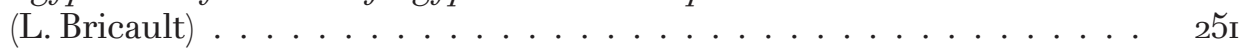

Helen VAN Noorden

Playing Hesiod. The 'Myth of the Races' in Classical Antiquity

(D. Bonanno)

Résumés . . . . . . . . . . . . . . . . . . . . . . . . . . . . . . . 257

Index .......................... . . 265 


\section{Actualités et débats}



Anabases 26 (2017), p. 177-181.

\section{Antiquités parallèles (7) La starlette des bandelettes}

Claude AZIZA

À l'heure où vous lirez ces lignes, vous aurez sans doute vu, à l'écran et en DVD, La Momie, d'Alex Kurtzman, sorti en juin 2017, qui raconte le réveil d'une maléfique reine. Bonne occasion pour revenir sur le thème.

La malédiction des momies, la vengeance des pharaons, le mystère des pyramides, autant de titres à sensation qui ( $\left.\mathrm{s}^{\prime}\right)$ éclatent à la Une des journaux et sur les affiches bariolées des films à frissons. Si l'Égypte fait rêver, ses momies font trembler. On ne les voit qu'à travers le filtre hollywoodien des années 30. Cet âge d'or du fantastique dont on devine vaguement qu'il a quelque chose à voir avec la crise économique de 1929 et, dans le cas de notre momie, avec la découverte, en 1922, du tombeau de Toutankhamon par Howard Carter (1874-1939). Avec plus de culture on remontera à ce fameux Roman de la momie, en reliant ce roman de Gautier et de 1857 à l'égyptomanie qui fit rage en France et en Europe, après la fameuse expédition d'Égypte.

Des follicules...

Bref, la momie ne demandait qu'un coup du destin pour s'ancrer dans l'espace imaginaire occidental, celui du roman qui venait de naître. Dès lors, une première veine, dont Gautier s'était fait le pionnier, est fondée, quasi-invariablement, sur la découverte d'une momie (le plus souvent du sexe féminin) dont l'archéologue (le plus souvent du sexe masculin) tombe amoureux et qu'il veut, contre toute raison, arracher à son sommeil. Dans cette femme, d'autant plus idéale qu'elle a bravé le temps pour venir d'un passé reculé, on aura reconnu une nostalgie des origines. Il s'agit essentiellement, à travers un amour qui tombe souvent dans le fétichisme du corps morcelé, cher aux hommes du XIX siècle, d'un déplacement du présent, 
celui de l'archéologue, dans le passé, celui de la momie. Mais le romancier sait bien que son héros qui viole le repos des morts en s'aventurant là d'où il n'est pas possible de revenir, brave des forces obscures. D'où son destin tragique, qu'on retrouvera tout au long des romans du $\mathrm{XX}^{\mathrm{e}}$ siècle. Un seul exemple : Le Basalte bleu (1927, TR, 1953, Le Livre de Poche), un roman de John Knittel (1891-1970), qui réécrit l'histoire de Howard Carter, où se mêlent fascination et impossibilité d'y succomber, devant une femme du passé, ramenée à la vie par un savant.

Mais le chef d'œuvre du genre reste La Momie (1989) d'Anne Rice (TR, 1992, Pocket, Terreur, $n^{\circ}$ 9076). On y trouve non pas une mais deux momies, ce qui est rarissime. Ensuite ces deux momies viennent de deux époques différentes. L’une, celle de Ramsès, remonte à la XIX ${ }^{\mathrm{e}}$ dynastie (Ramsès a régné de 1304 à 1237 av. J.-C.), l'autre, celle de Cléopâtre, à la dynastie grecque des Lagides et au Ier siècle avant notre ère. Autre originalité, ces deux momies ne sont pas anonymes ou quasi-imaginaires, comme la Tahoser de Gautier. Si la double intrigue amoureuse du roman reste dans le registre traditionnel, celui de l'amour fou, apparaissent, pourtant, des sentiments généralement inconnus de ce type de romans: la jalousie, la haine, le désir de vengeance, ainsi que le traitement d'un thème vieux comme le monde, celui de la quête de l'immortalité. Ce qui était au point de départ un fantasme d'archéologie - et le reste la plupart du temps d'ailleurs - a été ici magnifié par le génie d'une romancière de talent, qui a su retrouver les grands thèmes du fantastique. Mais d'autres romans présentent un schéma exactement inversé. Il ne s'agit plus du présent qui se plonge dans les troubles délices du passé pour vivre un amour qui brave les siècles, mais du passé qui vient troubler le présent, le plus souvent en la personne d'une momie-homme qui retrouve dans une femme d'aujourd'hui la figure - ou la réincarnation - de celle que jadis il aima. Là aussi, la mode a son mot à dire, et la fréquence de tels romans, à la fin du XIX ${ }^{\mathrm{e}}$ siècle et autour des années 1930, est là pour rappeler que le réalisme puis le naturalisme ont succédé au romantisme. Et qu'après Champollion est venu - ou viendra - Carter.

Passons rapidement sur Petite discussion avec une momie (1845), une nouvelle où Edgar Poe (1809-1849), avant Gautier, annonçait en mineur - sur un mode humoristique qui ne sera pas repris - le second thème. Sa momie est là pour prouver imperturbablement que le progrès n'existe pas et que, depuis quelques millénaires, les hommes n'ont rien inventé de nouveau.

En fait, c'est dans Le Joyau des sept étoiles (1903) que joue à plein le thème de la vengeance du passé. Son auteur, Bram Stoker (1847-1912), avait déjà créé, en 1897, avec Dracula, un nouveau type de roman, il en applique ici les règles. Ce mélange tout particulier d'archéologie et de terreur, déjà illustré dans un roman 
de Guy Boothby (1867-1905), Pharos l'Égyptien (1899), montre bien le danger, pour la société d'aujourd'hui, de jouer avec les démons égyptomaniaques (on trouvera ces deux romans dans le recueil : La Malédiction des momies, Fleuve Noir, 1997). Il n'est pas pensable d'énumérer tous les récits qui, désormais, font leurs délices de l'Égypte antique et de ses momies. Qui continuent d'être à la mode. Au point que, le 3 janvier 1907, le Tout-Paris se presse au Moulin-Rouge pour assister à une pantomime intitulée Rêve d'Égypte. On y voit une momie qui lascivement se débandelette, tendrement enlacée et embrassée par son amant. Ceci n’aurait rien que de très exotique, si derrière la momie ne se cachait la jeune Colette et, derrière le séducteur, Mathilde de Morny, marquise d'Elbeuf, descendante de Louis XV, arrière-petite-fille de Joséphine de Beauharnais et de Talleyrand, Missy pour les intimes, qui entretient avec Colette une scandaleuse et brûlante liaison. Comme quoi être une momie mène à tout, il suffit d'être extirpée de ses bandelettes...

Ainsi donc, à la différence des écrivains de la première partie du XIX ${ }^{\mathrm{e}}$ siècle, pétris d'égyptologie, une autre génération, plus tardive, a renoncé à se faire archéologue pour tenir un discours destructeur sur l'histoire. Revenir aux forces obscures de la nuit, c'était renoncer à la science pour un nouvel âge des ténèbres. Il ne semble pas qu'on en soit aujourd'hui sorti...

... aux pellicules

Le cinéma vient à peine de naître en 1895, qu'il se plonge dans les tombes égyptiennes : plus de 50 films, entre 1899 et 1927, où apparaissent des momies, au premier comme au second plan. Tout commence, en 1899, par le pillage de la tombe de Cléopâtre, filmé par Georges Méliès (1861-1938), dans Cléopâtre. De sa momie profanée et déchiquetée par les pilleurs sacrilèges s'échappe une fumée qui semble donner naissance à un fantôme vengeur.

Dès 1908, avec, en France, La Momie, une production Pathé et, aux États-Unis, The Princesse in the Vase, peut-être inspiré de loin par une nouvelle de Gautier (Le Pied de momie, 1840), un film de Billy Bitzer, l'intrigue s'étoffe et la durée s'allonge. Mais il faudra attendre l'année 1911 pour voir apparaître simultanément sur les écrans européens et américains, deux films importants qui abordent des thèmes que le parlant développera ensuite: La Momie d'Henri Desfontaines (1876-1931) et Le Roman de la momie d'Albert Capellani (1870-1931) adaptent, le premier, la nouvelle de Poe, le second, le roman de Gautier.

En 1932, au début du parlant, la momie apparaît enfin dans toute sa majesté. Quelques chiffres : entre 1932 et 2017, plus d'une centaine de films où elle batifole. Pourtant la chronologie est trompeuse, qui nous laisse croire à un flux continu alors que le thème fleurit par cycles. Le premier, le plus célèbre, sinon 
le plus connu aujourd'hui, regroupe la production de la firme Universal, entre, disons, 1932 et 1955. Dont son chef d'œuvre, La Momie de Karl Freund (DVD), avec Boris Karloff, qui raconte une histoire, maintes fois reprise, sous une forme ou sous une autre. La momie se nomme Im-ho-tep, nom - on s'en souvient - du célèbre médecin et architecte qui a bâti la pyramide à degrés de Djeser, sous la III è dynastie. L'hérö̈ne, princesse aimée puis perdue, a pour nom : Ank-es-enAmon, qui est historiquement une fille d'Akhenaton et de Néfertiti et l'épouse de Toutankhamon. L'intrigue est classique : l'imprudente lecture du Livre de Thot ramène à la vie la momie d'Im-ho-tep qui va découvrir sous les traits d'une jeune Américaine, Helen Grosvernor, sa princesse perdue.

Mais l'intérêt est ailleurs : dans le désir d'ancrer dans une Égypte pseudohistorique, au prix d'une chronologie acrobatique, une histoire d'amour telle que les romans cités plus haut nous l'ont contée. Cet habillage prisunico-égyptologique doit beaucoup, on s'en doute, à l'affaire Toutankhamon et à la “ malédiction » qui s'attache aux visiteurs du tombeau. Si la geste de la momie a débuté en même temps que celle des autres monstres, comme une sorte d'exorcisme à la crise économique de 1929, l'après-guerre apportera une autre sorte de terreur, celle du péril atomique, qui va refaire sortir, le plus souvent en technicolor, la momie de son tombeau.

Dès la fin des années 1950, la Hammer britannique a remplacé peu à peu l'Universal américaine. Elle se lance dans la réécriture des grands mythes de l'âge d'or, avec ses acteurs fétiches, Christopher Lee et Peter Cushing et ses réalisateurs habituels, Terence Fisher (1904-1980) surtout, mais aussi Michael Carreras (19271994) et John Gilling (1912-1985). Dracula, le monstre de Frankenstein, le loupgarou et, bien sûr, la Momie reviennent à l'écran.

La Malédiction des Pharaons (mais le titre original est : The Mummy) oppose, en 1959, Christopher Lee (Kharis/La Momie) qui a retrouvé en Isabelle (Yvette Furnaux) la princesse Ananka et Peter Cushing (DVD). Le film combine la version de 1932 et celles des années 1940, en y ajoutant les épices de la malédiction des pharaons : mutilation de Kharis, engloutissement de la momie dans les marais, sauvetage in extremis de l'héroïne, rien n'y manque, l'horreur en plus, car le cinéma commençait à se tourner vers le gore. Dans Les Maléfices de la momie (Michael Carreras, 1964, DVD), l'amour a disparu, remplacé par la vengeance de la momie, devenue Ra-Autef, un prince assassiné par son frère. Dans les Griffes de la momie (John Gilling, 1967, DVD) a des aspects particulièrement sanglants : la momie Prem, autrefois serviteur du pharaon Kah-to-bé, cherche à se venger des profanateurs.

Dans les années 1970 le danger atomique s'éloigne peu à peu et le moment est propice à un resourcement du thème. Ces années-là, nos monstres hanteront 
désormais plus la télévision que les cinémas. Parfois un sourire égaie le sujet, comme dans le délirant Papa Momie (William Dear, TV, 1985, DVD) où un acteur qui joue la momie dans un film de terreur et dont l'épouse est sur le point d'accoucher s'en va la retrouver sans avoir eu le temps de quitter ses bandelettes et se trouve poursuivi par les gens du coin, tandis que la vraie momie prend sa place et cabotine sous les caméras !

En 1997, on trouve encore une nouvelle adaptation du roman de Bram Stoker : La Légende de la momie, par Jeffrey Obrow (DVD) et sa suite (La Légende de la momie 2, par David Decoteau, DVD), enfin en 1998, La Malédiction de la momie par réalisateur Russel Mulcahy (DVD). Mais un an plus tard, le mythe allait être totalement renouvelé.

La Momie : le titre est simple et éloquent. Le réalisateur, Stephen Sommers, est l'un des plus talentueux spécialistes du film d'horreur. Il ressuscite donc, en 1999 (DVD), La Momie et, devant son succès, lui donne une suite (DVD), en 2001 : Le Retour de la Momie. Quatre films, tous en DVD, dans la foulée, prennent le relais et font revivre les aventures antiques du Roi Scorpion (C. Russel, en 2001, R. Mulcahy en 2008, R. Reiné en 2011 et M. Elliott en 2015).

Mais la famille des O'Connell reprend du service dans La Momie : La Tombe de l'empereur dragon (Rob Cohen, 2008, DVD). La nouveauté de ces trois Momies, ce sont d'abord des effets époustouflants - numérique oblige - mais surtout la transformation d'une aventure archéologique solitaire en une saga familiale, avec papa, maman, un gamin débrouillard mais bien mal élevé et un beau-frère coincé entre le désir de pantoufler et celui de chasser le trésor. C'est qu'entre temps les jeux vidéo ont fait leur apparition et que la momie ne fait plus peur : elle n'est que le prétexte à des cabrioles amusantes qui font sourire plutôt que de frissonner.

Plus d'amour éternel par-delà la barrière du temps, ou si peu, plus de malédiction venue du fond des âges, ou si pâle. La Momie est devenue alors, au prix d'un troublant streap-tease publicitaire, l'emblème d'un chewing-gum à la fraîcheur salvatrice qui autorise -enfin ! - le baiser. Sans risque...

Plus de bandelettes, à peine une starlette.

\section{Claude Aziza}

Université de la Sorbonne Nouvelle, Paris III claude.aziza@laposte.net 
